J. Life Earth Sci., Vol. 8: 31-39, 2013

ISSN 1990-4827

http://banglajol.info.index.php/JLES

(C) 2013, JLES, RU

\title{
PARENTAL ACCEPTANCE AND ACADEMIC ACHIEVEMENT OF TRIBAL AND NON-TRIBAL CHILDREN OF BANGLADESH
}

\author{
Rumana Aktar ${ }^{*}$, Mohd. Ashik Shahrier and Md. Mahabubur Rahaman Hridoy \\ Department of Psychology, University of Chittagong, Chittagong \\ *Corresponding author: e-mail: rumanarafi85@gmail.com
}

\begin{abstract}
The present study designed to explore the relationship between parental acceptance and academic achievement of tribal and non tribal children of Bangladesh. For this purpose, Bangla Version (Uddin, 2011) of PARQ/CQ (Short Form) for mother and father (Originally by Rohner, 2005) was administered on 96 respondents (48 tribal and 48 non-tribal) selected purposively from Khagrachari district, Bangladesh. Results indicated significant negative correlations between maternal acceptance scores and academic achievement scores and between paternal acceptance scores and academic achievement scores. Furthermore, results revealed significant differences in maternal acceptance, paternal acceptance and academic achievement between tribal and non-tribal children. From the results parental acceptance (maternal and paternal acceptance) found to be a stronger predictor of academic achievement where maternal acceptance created $8.3 \%$ variations and paternal acceptance created $10.3 \%$ variations in academic achievement of tribal and non-tribal children.
\end{abstract}

Key words: Maternal acceptance, paternal acceptance, academic achievement, children, ethnicity.

সারাংশ ঃ বাংলাদেশের উপজাতি ও অ-উপজাতি শিশুদের মা-বাবা কর্তৃক গ্রহণযোগ্যতা ও শিক্ষাক্ষেত্রে কৃতির মধ্যে সম্পর্ক অনুসন্ধানের জন্য বর্তমান গবেষণাটি পরিচালিত হয়েছিল। এ উদ্লেশ্যে শিশুদের উপযোগী করে তৈরি মা-বাবা কর্তৃক গ্রহণযোগ্যতা -প্রত্যাখ্যান মাণক (PARQ/CQ, Short Form) এর বাংলা অনুবাদ (উদ্দিন, ২০১১) বাংলাদেশের খাগড়াছড়ি জেলা হতে উদ্দেশ্যমূলকভাবে নির্বাচিত ৯৬ জন শিশ্ (৪b- জন উপজাতি, ৪b- জন অ-উপজাতি) এর উপর প্রয়োগ করা হয়েছিল। ফলাফলে দেখা যায়, মা কর্তৃক গ্রহণযোগ্যতা সাফল্যাংক ও শিক্ষাক্ষেত্রে কৃতি সাফল্যাংক এবং বাবা কর্তৃক গ্রহণযোগ্যতা সাফল্যাংক ও শিক্ষাক্ষেত্রে কৃতি সাফল্যাংকের মট্যে তাৎপর্যপর্ণ বিপরীতমূখী সহসম্পর্ক বিদ্যমান। ফলাফলে আরও দেখা যায়, মা কর্তৃক গ্রহণযোগ্যতা, বাবা কর্তৃক গ্রহণযোগ্যতা এবং শিক্ষাক্ষেত্রে কৃতি এর ক্ষেত্রে উপজাতি ও অ-উপজাতি শিফ্ডের মট্যে তাৎপর্যপূর্ন পার্থক্য বিদ্যমান। এছাড়াও ফলাফলে উপজাতি ও অ-উপজাতি শিশুদের মা-বাবা কর্তৃক গ্রহণযোগ্যতা শিক্ষাক্ষেত্রে কৃতিত্বে তারতম্য সৃষ্টির ক্ষেত্রে গুরুত্বপূর্ণ প্রভাবক হিসাবে কাজ করে যেখানে মা কর্তৃক গ্রহণযোগ্যতা শিক্ষাক্ষেত্রে কৃতিত্বের ক্ষেত্রে ৮-৩\% এবং বাবা কর্তৃক গ্রহণযোগ্যতা শিক্ষাক্ষেত্রে কৃতিত্তের ১০.৩\% তারতম্য সৃষ্টিতে সক্ষম।

\section{Introduction}

Children everywhere need a specific form of positive response i.e. acceptance from parents and other attachment figures. When this need is not met satisfactorily, children worldwide - regardless of variations in culture, gender, age, ethnicity or such other defining conditions, tend to report themselves to be hostile and aggressive, dependent or defensively independent, over impaired in self- adequacy and selfesteem and to have a negative worldview among other responses. Rohner (Rohner \& Khaleque, 2006; Rohner, Khaleque \& Cournoyer, 2007, 2012) formulated the Parental Acceptance-Rejection Theory (PAR Theory) which is robust and used worldwide regardless of differences in culture, race, gender, geographic context, or such defining conditions. The Parental acceptancerejection theory (PAR Theory) is an evidence-based theory of socialization and lifespan development that seeks to predict and explain major causes, consequences, and other correlates of parental acceptance and rejection worldwide.

The form of communication between a parent and a child has a reciprocal effect (Russo \& Owens, 1982). Parents basically mold and shape their children into adults through their world of influence (Baumrind, 1971). A way of reflection between parent and child relationships is parenting and it is a complex activity that includes many specific attitudes and behaviors that work separately and collectively to influence child outcomes and generate an emotional bond in which the parent's behaviors are expressed (Darling \& Steinberg, 1993; Darling, 1999). Parenting can be explained in terms of two components such as parental responsiveness and demandingness (Fletcher et al., 2008). Parental demandingness is the extent to which parents set guidelines for their children, and how their discipline based on these guidelines. Parental responsiveness is the emotional characteristic of parenting. Responsiveness passes on to the degree to which parents support their children and attend their children's needs. Both parenting responsive and demanding has been linked to secure attachment in children (Karavasilis, Doyle \& Markiewicz, 2003). Fletcher et al. (2008) argued that when parents scored low on both dimensions of responsiveness and demandingness then children demonstrated the most problematic development and caused internalizing, externalizing, and social problems.

Together parental acceptance and rejection form the warmth dimension of parenting. This is a dimension or continuum on which all humans can be placed because everyone has experienced in childhood more or less love at the hands of major caregivers. Thus, the warmth dimension has to do with the quality of the affection bond between parents and their children, and with the 
physical, verbal, and symbolic behaviors parents use to express these feelings. One end of the continuum is marked by parental acceptance, which refers to the warmth, affection, care, comfort, concern, nurturance, support, or simply loves that children can experience from their parents and other caregivers. The other end of the continuum is marked by parental rejection, which refers to the absence/significant withdrawal of these feelings and behaviors, and by the presence of a variety of physically, and psychologically hurtful behaviors and affects. Parents may neglect their children as a way of trying to cope with their anger toward them. Neglect is not simply a matter of failing to provide for the material and physical needs of children, however; it also pertains to parents' failure to attend appropriately to children's social and emotional needs. Often, for example, neglecting parents pay little attention to children's needs for comfort, solace, help, or attention; they may also remain physically as well as psychologically unresponsive or even unavailable or inaccessible. All these behaviors, real or perceivedindividually and collectively-are likely to induce children to feel unloved or rejected. Extensive crosscultural research over the course of half a century in PAR Theory reveals that parental rejection can be experienced by any combination of four principal expressions: (1) cold and unaffectionate, the opposite of being warm and affectionate, (2) hostile and aggressive, (3) indifferent and neglecting, and (4) undifferentiated rejecting. Undifferentiated rejection refers to individuals' beliefs that their parents do not really care about them or love them, even though there might not be clear behavioral indicators that the parents are neglecting, unaffectionate, or aggressive toward them (Rohner, Khaleque \& Cournoyer, 2012).

In effect, much of parental acceptance-rejection is symbolic (Kagan, 1974, 1978). Therefore, to understand why rejection has consistent effects on children and adults, one must understand its symbolic nature. Certainly in the context of ethnic and crosscultural studies investigators must strive to understand people's symbolic, culturally-based interpretations of parents' love-related behaviors if they wish to fully comprehend the acceptance-rejection process in those settings. That is, even though parents everywhere may express, to some degree, acceptance (warmth, affection, care, concern) and rejection (coldness, lack of affection, hostility, aggression, indifference, neglect), the way they do it is highly variable and saturated with cultural or sometimes idiosyncratic meaning. For example, parents anywhere might praise or compliment their children, but the way in which they do it in one socio- cultural setting might have no meaning (or might have a totally different meaning) in a second setting.

On the issue of academic achievement, McClelland's (1961) early work on the emergence of the achievement motive suggests that parental attitudes can foster school identification by placing emphasis on academic success and independence. It was also hypothesized in a study conducted by Vierstein \& Hogan (1975) that high achievement motivation in boys can be explained in terms of effective socialization to norms of achievement in which the desire to achieve is enhanced by a stable, harmonious home environment supplied by parents with similar values, by the presence of an acceptable parental model and by extra encouragement provided by an achievement oriented maker. Academic achievement is generally measured on the basis of examination scores or by standardized test scores. According to Good (1993) Academic achievement means knowledge attained or skills developed in the school subjects, usually designated by test scores or by marks assigned by teachers or by both.

Parent-child interaction has long been considered to have a crucial influence on a child's academic performance and development (Prindle \& Resinski, 1989; Van Meter, 1994; Barnard \& Kelly, 1990). Children's higher school achievement rates, higher attendance rates, lower delinquency and dropout rates, and increased high school completion rates are enhanced by a strong parent-child relationship (Ziegler, 1987). A number of studies have reported that a warm and responsive parent-child interaction is positively related to self-esteem, social acceptability, and achievement in young children (Estrada, Arsenio, Hess \& Holloway, 1987; Bradley, Caldwell \& Rock, 1988). Researchers have reported that parent-child interactions, specifically stimulating and responsive parenting practices, are important influences on a child's academic development (Christian, Morrison, \& Bryant, 1998; Committee on Early Childhood Pedagogy, 2000).

Kamble and Adsul (2012) aimed at investigating the effect of parental acceptance and academic climate on academic performance of adolescents. 180 students studying at 10th class in various schools of Sangli and Kolhapur districts of Maharashtra State, India were selected by random sampling method. 2 X 2 factorial research design was employed. Two- way ANOVA was used to analyze the collective data. The findings of the study revealed that parental acceptance and academic climate strongly and significantly affects academic performance of adolescents. Khan et al. (2010) 
investigated the effects of perceived teacher acceptance as well as perceived maternal and paternal acceptance on the academic achievement and school conduct of 362 seventh-grade adolescents in the Mississippi Delta region of the United States. Results showed a significant correlation between perceived teacher acceptance and boys' (but not girls') overall grade point average (GPA). Perceived maternal acceptance (but not paternal acceptance) also correlated with boys' GPA. Neither maternal nor paternal acceptance, however, was correlated with girls' academic achievement. A study (Topor et al., 2010) on parent involvement and student academic performance was conducted using a sample of 158 seven-year old participants, their mothers, and their teachers. Results indicated a statistically significant association between parent involvement and a child's academic performance, over and above the impact of the child's intelligence.

Elias (2006) investigated a slightly different but related issue in Bangladesh on 100 students (50 from fourth and 50 fifth grades). Result showed no significant relations between parent-child interaction and academic achievement. A study carried out in the Mississippi Delta Region of the United States on 362 seventh grade adolescents reported that the perceived maternal acceptance was significantly correlated with boys' GPA but neither maternal nor paternal acceptance was correlated with girls' academic achievement (Khan, Haynes, Armstrong \& Rohner, 2010). Uddin (2011) explored the relation between parental warmth and academic achievement in Bangladesh on 300 students of four schools of capital city Dhaka. The result revealed that both maternal and paternal warmth were positively related to academic achievement of children. Heaven and his colleagues (2002) conducted a study on family relationship, school climate and personality and revealed that the personality traits were consistently related to attitudes to school and the parental factors (care and overprotection). Stephen J. Ceci et al. (1997) proposed that parental acceptance lead to academic performance. Children who are successful and well developed came from families where positive relationship existed between them and their parents, where as children, who were discouraged and rejected at home they fail to get success in academic achievement (Bowlby, 1967). Several other researchers concluded that, parental acceptance has significant impact on academic performance of adolescents (Epstein, 1989; Hoover - Dempsey and Sandler, 1997; Rayn and Adams, 1995).
Kazmi, Sajjid, and Pervez (2011) study explored impact of father's style of dealing with their children at home and their academic achievements at school and result were in favor of the fathers' involvement for the academic achievements. In a meta-analysis containing 21 studies, the impact of parental involvement on the academic achievement of minority children was significant for all marginal groups. For all the groups, parental involvement as a whole, affected all the academic variables by at least two tenths of a standard deviation unit. However, the results indicated that parental involvement affected the academic achievement of minority students (Jeynes, 2003). Xitao and Michael (2001) found parental involvement as positively related to students' academic achievement. Luby et al. (2012) found that the early experience of maternal nurturance among preschoolers was strongly predictive of larger hippocampus volume among the same children at school age. These results are important because the hippocampus is a region of the brain that is central to memory, emotion regulation, stress modulation, and other functions - all of which are essential for healthy socio-emotional adjustment. In a study of Kordi and Baharudin (2010), the contributions of parenting attitude and style were examined in relation to children's school achievement. Findings revealed a strong relationship between children's school achievement and parenting attitude and style.

The primary census report of Bangladesh, 2011 illustrates that total ethnic population group of Bangladesh is 27. Major ethnic groups of Bangladesh are: The Bangalees, The Chakmas, The Santal, The Tanchangya, The Tippearas, The Mros, Khasis, Garas, Khajons etc. Bangalees are the largest ethnic group in Bangladesh, forming more than $98 \%$ of the entire population. Except Bangalees, the other ethnic groups are known as Tribal group in Bangladesh. A sense of ethnic identity becomes salient for many ethnic minority adolescents as they explore the significance of their ethnic group membership in defining who they are (Spencer \& Markstrom-Adams, 1990; Phinney, 1990). Bangladesh is a densely populated country of South East Asia that has a rich tribal presence. There are about 58 tribes living in different parts of the country. Bangladesh has 1.2 million tribal people, which is just above 1 percent of the total population (Mullah, Parveen \& Ahshanullah, 2007). Major tribes of this country live in Chittagong Hill tracts and in the regions of Mymensingh, Sylhet, and Rajshahi. Whatever may be the population of them, they differ with the majority Bangalee ethnic group with respect to socio-cultural set up, marital customs, rites and rituals, food habits, 
behaviour and life styles, school environments, commitment to school, child rearing and parenting styles, parental supervision, family climate, parental attachment \& bonding, parental education, community support and other basic needs. Based on these differences and by considering the above mentioned literature reviews, an attempt has been made in this study to see the relationship between parental (maternal\& paternal) acceptance and academic achievement of tribal (Chakma, Marmas etc.) and non tribal (Bangalee) children of Bangladesh.

\section{Objectives of the Study}

The main objective of the study was to explore the relationship between parental (paternal and maternal) acceptance and academic achievement of tribal and non tribal children of Bangladesh. Some specific objectives of the study are as follows:

1. To determine the differences in parental (paternal and maternal) acceptance and academic achievement with reference to ethnicity.

2. To determine how much variation as a predictor parental acceptance (maternal \& paternal) can create in explaining ethnic adolescents' academic achievement.

\section{Rationale of the Study}

Family is the primary agent of socialization of children. The most important relationship in a family is the stable and healthy parent-child relationship that facilitates effective socialization and proper adaptation of a child. On the one end of parent-child interaction is parental acceptance and on the other end is parental rejection. Parents' acceptance or rejection behaviors have an important impact on children's every sphere of lives especially in their academic pursuits (e.g. Uddin, 2011; Estrada, Arsenio, Hess \& Holloway, 1987; Bradley, Caldwell \& Rock, 1988). In the multiethnic culture of Bangladesh, it is deeply observed that there remain various socio-cultural aspects believed to create differences between tribal (Chakma, Marma, Santal etc.) and non-tribal (Bangalees) children of Bangladesh. The socio-economic set up, customs, rituals, food habits, life styles, language, school culture, parenting styles, parental attitudes towards children, parental attachment and bonding, parental monitoring, education, social and academic feedbacks etc. believed to vary greatly among tribal and non-tribal children of Bangladesh. Obviously there may be variations between tribal non tribal children in parent child interactions or parental attitudes because these two groups are reared in different socio-cultural set up. So, parental attitudes, parental attachment, parenting styles in a cultural setting of one group seems to be evaluated negatively by members of other group because these attitudes or behaviors are regarded as negative in latter group's cultural \& societal codes or norms and vice versa. In spite of this, with respect to the overall socio cultural context of Bangladesh, some common patterns of behaviour and life styles exist and along with it, the perception of children that whether they are being accepted or rejected by their parents may be the determinant of parental acceptance or rejection. These acceptance (warmth, affection, care, comfort, concern, nurturance, support) or rejection(hostility, aggression, neglect or psychologically hurtful behaviors) attitudes of parents in turn may determine children's high or low commitment to school, high or low academic performance, school dropout rates, high or low self regard, self esteem, self efficacy and their good or poor mental well being. By considering these factors this study makes an attempt to explore the relationships between parental acceptance and academic achievement of tribal and non-tribal children of Bangladesh. And it is hoped that this study will enhance the domain of PAR theory cross culturally and the outcome of this study will be beneficial for different children welfare based NGO's to play vital role in awaking parents about the importance of parent-child interaction and in arranging effective programs for prevention of school failure and dropout rates of children for ensuring a better future of them.

\section{Materials and Methods}

Sample: Two stage sampling were used for the present study.

School Selection: Purposively three schools were selected from Khagrachhari District.

Respondents Selection: Initially the sample of the study consisted of 104 respondents. Among them, respondents showing perceived parental acceptance were 96 and respondents showing perceived parental rejection were 08 . As our study concerned with parental acceptance so we considered only the data of those respondents showing perceived parental acceptance. Thus, finally the sample of the study consisted of 96 students of $6^{\text {th }}$ and $9^{\text {th }}$ grades. Half of them were tribal (Chakma \& Marmas) and the rest half were non-tribal (Bangalee) children. Their ages ranged from 10 to 14 years. Each group was equally divided into tribal and non tribal on the basis of ethnicity $(\mathrm{N}=48$ for each group).Then they were divided into $6^{\text {th }} \& 9^{\text {th }}$ grades in terms of grade ( $\mathrm{N}=24$ for each group). Again they were subdivided into male and female in terms of gender ( $\mathrm{N}=12$ for each group).They were selected purposively from three schools of Khagrachhari District of Bangladesh. The sample distribution is given below: 
Table 1 Distribution of respondents according to ethnicity (Tribal\& Non-Tribal), grade $\left(6^{\text {th }} \& 9^{\text {th }}\right)$ and Gender (Male \&Female)

\begin{tabular}{cccccc}
\hline Variables & \multicolumn{2}{c}{ VI } & \multicolumn{2}{c}{ IX } & Total \\
& Male & Female & Male & Female & \\
\hline Tribal & 12 & 12 & 12 & 12 & 48 \\
Non-tribal & 12 & 12 & 12 & 12 & 48 \\
Total & 24 & 24 & 24 & 24 & 96 \\
\hline
\end{tabular}

Instruments

Personal Information Form (PIF): The PIF elicited demographic, personal and social information that included respondents' gender, age, GPAs in PSC/JSC Exams, number of siblings, birth order, family size, parental education, parental occupation, ethnicity, religious affiliation etc.

Bangla Version (Uddin, 2011) of the Child PARQ/CQ: Mother and Father Versions (Short Form) Originally Developed by Rohner (2005) : Child Parental Acceptance Rejection Questionnaire (PARQ/CQ): Mother and Father Versions (Short Form) were used to assess children's perceptions of parental (maternal and paternal) acceptance and rejection. This form contains 29 items. Out of 29 items, 24 items encompassed the four different dimensions of Parental Acceptance-Rejection Questionnaire (PARQ) and the remaining 5 items represented the Control subscale. Respondents were instructed to ask themselves if an item is basically true or untrue about the way their parents treat them. If the statement is applied to them, they were instructed to score themselves, "Is it almost always true?" Or "Is it sometimes true?" Or if an item did not apply to the way their parents treated them, they were instructed to ask themselves, "Is it rarely true?" Or "Is it almost never true?" Numerical score for each response was recorded as 4 for "almost always true", 3 for "sometimes true", 2 for "rarely true", and 1 for "almost never true. In order to minimize yay/nay-saying (Couch \& Kenston, 1960) and other forms of responseset bias, nearly half (9 out of 24) of the items on the PARQ (short form) must be reverse scored to create a total acceptance rejection score. More specifically, the entire warmth/affection scale must be reverse scored to create the form of rejection called coldness/lack of affection. Only one of the items (item 18) of the indifference/neglect items is reverse scored. Reverse scoring items this way allows the transformed warmth/affection scale to be added to the other three rejection scales, thus creating an overall measure of perceived acceptance-rejection. The warmth/affection scale refers to parent-child relationships where parents are perceived to give love or affection without qualification. Eight items included in this subscale are $1,4,11,15,21,23,27$, and 29. Scores on this subscale spread from a low of 8 (maximum acceptance) to a high of 32.The hostility/aggression scale refers to conditions where children believe their parent is angry, bitter, or resentful of them (i.e., perceived hostility) or to conditions where children believe their parent intends to hurt them physically or verbally (perceived aggression). Six items included in this subscale are 5, 8, $12,17,22$, and 24 . Scores on this subscale spread from a low of 6 (maximum acceptance) to a high of 24. The indifference/neglect scale assesses conditions where children see their parent as unconcerned or uninterested in them. Six items included in this subscale are 2, 9, 13, 16,18 , and 24. Scores on this subscale spread from a low of 6 (maximum acceptance) to a high of 24 . Undifferentiated rejection refers to individuals' beliefs that parents do not really care about them or love them, even though there might not be clear behavioral indicators that the parents are neglecting, unaffectionate, or aggressive toward them. Four items included in this subscale are 6, 10, 19, and 24. Scores on this subscale spread from a low of 4 (maximum acceptance) to a high of 16 . Control refers to children's perceptions of the behavioral control (i.e., permissiveness or strictness) by their parents they now experience. Five items included in this subscale are 3, $7,14,20$, and 26. Scores on this subscale spread from a low of 5 (maximum acceptance) to a high of 20.The lowest possible score of this scale (for both mother and father versions) is 24 and highest possible score is 96 with a possible midpoint score of 60 . That means, scores at or above this midpoint represents more parental rejection as perceived by the offspring. The Lower the scores (Less than 60) the more will be the parental acceptance perceived by the offspring. The internal consistency reliability of the PARQ/CQ: Mother was .86 and for father was .84. Both mother and father versions of $\mathrm{PARQ} / \mathrm{CQ}$ finally proved to be psychometrically sound because of its satisfactory convergent validity. The PARQ has been used in over 400 studies worldwide and is known to have outstanding reliability and validity for use in crosscultural research (Khaleque \& Rohner, 2002; Rohner, 2005).

Academic Achievement: The academic achievement was measured from Grade Point Average (GPA) of respondents' previous class. For authentic measure, the GPAs of JSC and PSC were taken into consideration that was collected from respondents' school records. Here, higher GPAs indicated better academic achievement. 


\section{Procedure}

A written permission was received from the U.N .O. of Khagrachhari district for collecting data. The headmaster of each school was approached for his/her cooperation. All measures along with the PIF were administered to students in classroom settings. Before the administration, necessary rapport was established with respondents. Respondents were asked to complete the questionnaires at their own pace. Respondents were assured that their responses will be kept confidential and that there is nothing like right or wrong responses to any question. Finally, respondents were encouraged to ask questions coming in their minds during the task and they were informed of their right to withdraw from the study at any time. It took half an hour on average to complete the task. On completion, every respondent was given a token gift with thanks for their participation in the study.

\section{Design of the Study}

Cross sectional survey design was used for the present study.

\section{Results}

Standardized statistical analyses were used to explain the collected data. The results and their interpretations are presented in the following section.

Table 2 Correlations between Maternal Acceptance and Academic Achievement of Tribal and Non-Tribal Children

\begin{tabular}{lll}
\hline Variables & $\begin{array}{l}\text { Maternal } \\
\text { Acceptance }\end{array}$ & $\begin{array}{l}\text { Academic } \\
\text { Achievement }\end{array}$ \\
\hline $\begin{array}{l}\text { Maternal Acceptance } \\
\text { Academic Achievement }\end{array}$ & $-.304(* *)$ & \\
\hline
\end{tabular}

**Correlation is significant at the 0.01 level (2-tailed)

Table 2 indicates a significant negative correlation between maternal acceptance and academic achievement scores of tribal and non-tribal children $(r=-.304$, $\mathrm{p}<0.01)$.That is, the lower the maternal acceptance scores (e.g. lower scores indicate more maternal acceptance), the higher will be the academic achievement scores (e.g. higher scores indicate high academic achievement) of tribal and non-tribal children and vice versa.

Table 3 Correlations between Paternal Acceptance and Academic Achievement of Tribal and Non-Tribal Children

\begin{tabular}{lll}
\hline Variables & $\begin{array}{l}\text { Paternal } \\
\text { Acceptance }\end{array}$ & $\begin{array}{l}\text { Academic } \\
\text { Achievement }\end{array}$ \\
\hline $\begin{array}{l}\text { Paternal Acceptance } \\
\text { Academic Achievement }\end{array}$ & $-.322(* *)$ & \\
\hline
\end{tabular}

**Correlation is significant at the 0.01 level (2-tailed)

Table 3 indicates a significant negative correlation between paternal acceptance and academic achievement scores of tribal and non-tribal children $(r=-.322$, $\mathrm{p}<0.01)$.That is, the lower the paternal acceptance scores (e.g. lower scores indicate more paternal acceptance), the higher will be the academic achievement scores (e.g. higher scores indicate high academic achievement) of tribal and non-tribal children and vice versa.

Table 4 Differences in Parental Acceptance (Maternal and Paternal Acceptance) and Academic Achievement of Tribal and Non-Tribal Children

\begin{tabular}{llcccccc}
\hline Variables & Ethnicity & $\mathrm{N}$ & $\mathrm{Mean}$ & $\mathrm{Sd}$ & $\mathrm{df}$ & $\mathrm{t}$ & $\mathrm{p}$ \\
\hline Maternal & Tribal & 48 & 45.10 & 7.470 & 94 & 5.114 & $0.001^{*}$ \\
Acceptance & Non-Tribal & 48 & 37.50 & 7.095 & & & \\
Paternal & Tribal & 48 & 45.19 & 7.347 & & & \\
Acceptance & Non-Tribal & 48 & 40.27 & 7.356 & 94 & 3.276 & $0.001^{*}$ \\
Academic & Tribal & 48 & 2.93 & 1.07 & 94 & 4.792 & $0.001^{*}$ \\
Achievement & Non-Tribal & 48 & 3.79 & 0.627 & & & \\
\hline
\end{tabular}

$\mathrm{P}<0.01 *$

Results reported in Table 4 revealed a significant difference $(\mathrm{df}=94, \mathrm{t}=5.114, \mathrm{p}<0.01)$ in maternal acceptance of tribal $(\mathrm{M}=45.10, \mathrm{Sd}=7.470)$ and nontribal $(\mathrm{M}=37.50, \mathrm{Sd}=7.095)$ children. That is, non-tribal children were more maternally accepted as compared to their tribal counterpart. Again, the results revealed a significant difference $(\mathrm{df}=94, \mathrm{t}=3.276, \mathrm{p}<0.01)$ in paternal acceptance of tribal $(\mathrm{M}=45.19, \mathrm{Sd}=7.347)$ and non-tribal $(\mathrm{M}=40.27, \mathrm{Sd}=7.356)$ children. That is, nontribal children were more paternally accepted as compared to their tribal counterpart. The results also showed a significant difference $(\mathrm{df}=94, \mathrm{t}=4.792$, $\mathrm{p}<0.01)$ in academic achievement of tribal $(\mathrm{M}=2.93$, $\mathrm{Sd}=1.07)$ and non-tribal $(\mathrm{M}=3.79, \mathrm{Sd}=.627)$ children. That is, non-tribal children showed higher academic achievement as compared to their tribal counterpart.

Table 5 Regression of Academic Achievement on Maternal Acceptance

\begin{tabular}{|c|c|c|c|c|c|c|c|}
\hline \multirow[t]{2}{*}{ Predictors } & \multicolumn{2}{|c|}{ Un std. Coeff. } & \multirow{2}{*}{$\begin{array}{c}\text { Std. } \\
\text { Coeff. } \\
\beta\end{array}$} & \multirow[t]{2}{*}{$\mathrm{t}$} & \multirow[t]{2}{*}{$\mathrm{P}$} & \multirow[t]{2}{*}{$r_{p}$} & \multirow[t]{2}{*}{$\mathrm{r}_{\mathrm{p}}^{2} \times 100$} \\
\hline & B & SE & & & & & \\
\hline (Constant) & 4.85 & 0.492 & & 9.87 & 0.000 & & \\
\hline $\begin{array}{c}\text { Maternal } \\
\text { acceptance }\end{array}$ & -0.036 & 0.012 & -0.304 & 3.09 & 0.003 & -0.304 & 9.24 \\
\hline
\end{tabular}

The value of standardized beta in table $5(\beta=-.304)$ reveals that the increases of 1 standard deviation unit in academic achievement, decreases 0.304 standard deviation unit in maternal acceptance. The value of adjusted $\mathrm{R}^{2}$ (Adjusted $\mathrm{R}^{2}=0.083,\left(\mathrm{~F}_{1,94}=9.59, \mathrm{P}<.01\right)$ ) in table-5 also reveals that the predictor variable or maternal acceptance explains $8.3 \%$ variance of criterion variable 'academic achievement'. Furthermore, part correlation coefficient in the above table indicates that 
the unique contribution of 'maternal acceptance' to explain the variance in academic achievement of tribal and non-tribal children was 9.24\%. Thus, maternal acceptance was a stronger predictor to create variations in tribal and non-tribal children's academic achievement.

Table 6 Regression of Academic Achievement on Paternal Acceptance

\begin{tabular}{cccccccc}
\hline Predictors & \multicolumn{2}{c}{ Un std. Coeff. } & $\begin{array}{c}\text { Std. } \\
\text { Coeff. }\end{array}$ & $\mathrm{t}$ & $\mathrm{P}$ & $\mathrm{r}_{\mathrm{p}}$ & $\mathrm{r}_{\mathrm{p}}^{2} \times 100$ \\
& $\mathrm{~B}$ & $\mathrm{SE}$ & $\beta$ & & & & \\
\hline $\begin{array}{c}\text { (Constant) } \\
\begin{array}{c}\text { Paternal } \\
\text { acceptance }\end{array}\end{array}$ & -0.095 & 0.535 & & 9.53 & 0.000 & & 10.37 \\
\hline
\end{tabular}

Adjusted $\mathrm{R}^{2}=0.103,\left(\mathrm{~F}_{1,94}=10.85, \mathrm{P}<0.01\right)$

The value of standardized beta in table $6(\beta=-.322)$ reveals that the increases of 1 standard deviation unit in academic achievement, decreases 0.322 standard deviation unit in paternal acceptance. The value of adjusted $\mathrm{R}^{2}$ (Adjusted $\mathrm{R}^{2}=0.103,\left(\mathrm{~F}_{1,94}=10.85, \mathrm{P}<.01\right)$ ) in table 6 also reveals that the predictor variable or paternal acceptance explains $10.3 \%$ variance of criterion variable 'academic achievement'. Furthermore, part correlation coefficient in the above table indicates that the unique contribution of 'paternal acceptance' to explain the variance in academic achievement of tribal and non-tribal children was $10.37 \%$. Thus, paternal acceptance was a stronger predictor to create variations in tribal and non-tribal children's academic achievement.

\section{Discussion and Conclusion}

The main objective of the present study was to explore the relationship between parental acceptance and academic achievement of tribal and non-tribal children of Bangladesh. Some specific objectives of this study were: to determine the differences in parental (paternal and maternal) acceptance and academic achievement with reference to ethnicity and to determine how much variation as a predictor academic achievement can create in explaining ethnic adolescents' parental (paternal and maternal) acceptance. Towards this end, Bangla Version (Both Mother and Father Versions) of PARQ/CQ (Uddin, 2011) was administered on 96 respondents (48 tribal and 48 non-tribals) of Khagrachari district, Bangladesh. Results of the study were analyzed through correlations, regressions and $t$ values.

Results of the study revealed significant negative correlations between maternal acceptance and academic achievement scores and also between paternal acceptance and academic achievement scores (Table 2
\& 3). That means, the lower the maternal acceptance scores (e.g. lower scores indicate more maternal acceptance), the higher will be the academic achievement scores (e.g. higher scores indicate high academic achievement) of tribal and non-tribal children and vice versa.

It was found from the results of the study that there were significant differences in maternal acceptance, paternal acceptance and academic achievement between tribal and non tribal children. That is, non tribal children were more maternally accepted, more paternally accepted and showed higher academic achievement as compared to their tribal counterpart (Table 4).

The value of adjusted $\mathrm{R}^{2}$ (Adjusted $\mathrm{R}^{2}=0.083$, $\left(\mathrm{F}_{1}\right.$, $\left.{ }_{94}=9.59, \mathrm{P}<.01\right)$ ) in table 5 revealed that the predictor variable 'maternal acceptance' explains $8.3 \%$ variance of criterion variable 'academic achievement'. Again, the value of adjusted $\mathrm{R}^{2}$ (Adjusted $\mathrm{R}^{2}=0.103,\left(\mathrm{~F}_{1}\right.$, $\left.{ }_{94}=10.85, \mathrm{P}<.01\right)$ ) in table 6 revealed that the predictor variable 'paternal acceptance' explains $10.3 \%$ variance of criterion variable 'academic achievement'. Thus, parental acceptance (maternal and paternal) was a stronger predictor to create variations in tribal and nontribal children's academic achievement. The results of the study are consistent with several previous studies (Estrada, Arsenio, Hess \& Holloway, 1987; Bradley, Caldwell \& Rock, 1988; Christian, Morrison, \& Bryant, 1998; Committee on Early Childhood Pedagogy, 2000; Kamble and Adsul, 2012; Khan et al., 2010; Topor et al., 2010, Uddin, 2011; Jeynes, 2003; Xitao and Michael, 2001; Luby et al., 2012).

The results of this study are also consistent with PAR theory's socio-cultural subsystems model and sub theory. This theory shows that parents' any given form of behaviour(acceptance or rejection) is shaped by the maintenance systems of a society including such social institutions as family structure, household organizations, economic organization, political organization, system of defense, and other institutions that bear directly on the survival of a culturally organized population within its natural environment (Kardiner,1939, 1945a, 1945b; Whiting \& Child ,1953; Bronfenbrenner,1979, 1994; Berry,2006; Rohner, 1986). Due to the poor maintenance systems of these social institutions in a tribal set up as compared to the socio-culturally enriched non-tribal set up, it is believed that the personal characteristics such as temperament, intelligence, academic achievement, behavioral dispositions and personality development are varied to 
a great extent between children of these two set ups. In a tribal set up, parents' social isolation without social and emotional supports, economic deprivation, appear universally to be at greatest risk for withdrawing love and affection from children. But in the non tribal set up, a social, emotional and economic support network help parents to accept their children more positively with warmth, love, affection and appreciation. Hence the non tribal children as compared to their tribal counterpart develop themselves through greater self confidence, self regard, self esteem and they possess high commitment to school, have lower dropout rates and are academically high achievers as compared to tribal children. Thus it is plausible to argue that there is a clear relation between academic achievement and parental acceptance of children. Similarly, ethnic differences due to the availability or non availability of various psychological and socio-cultural factors are also responsible to create differences in parental (maternal and paternal) acceptance and academic achievement of children of tribal and non-tribal set up. These sociocultural explanations in the perspective of PAR theory (e.g. Socio-cultural Subsystems Model and Sub Theory) are in line with the objectives of the present study and these explanations have clearly reflected in the results of the study.

\section{Concluding Remarks}

The significant findings of the study could not be emphatically generalized without substantial empirical researches in such areas including different ethnic groups' children in the context of Bangladesh. Here, the basic necessity for future research lies. Further research should be designed as to find out more variables like teacher acceptance- rejection, peer acceptancerejection etc. and their effects on the academic pursuits of different ethnic groups' children of Bangladesh. It can be argued that the problem of researching parental acceptance and academic achievement of tribal and non-tribal children is too vast to be dealt with in such a short research. However, it throws light into this area which needs further studies and exploration.

\section{References}

Barnard, KE \& Kelly, JA. 1990. Assessment of parent-child interaction. In Meisels, S.J. and Shonkoff, J.P (Eds.) Handbook of early childhood intervention (pp. 278-302) London: Cambridge University Press.

Baumrind, D.1971. Current patterns of parental authority. Developmental Psychology, 4(1),1-103

Berry, JW, \& Poortinga, YH. 2006. Cross-cultural theory and methodology. In J. Georgas, J.W Berry, F.J.R. vander Vijver, C. Kagitcibas, \& Y.H. Poortinga (Eds.), Families across cultures:
A 30-Nation Psychological Study (pp.51-71). Cambridge University Press.

Bowllby, TR. 1967. Personality roles in large family. Child Development, 26, 71-78.

Bradley, RH, Caldwell, BM, \& Rock, SL. 1988. Home environment and school performance: A ten year follow- up and examination of three models of environmental action. Child Development, 59, 852-867.

Bronfenbrenner, U. 1979. The ecology of human development: Experiments by nature and by design. Cambridge, MA: Harvard University Press.

Bronfenbrenner, U. 1994. Ecological models of human development. In International Encyclopedia of Education, Vol. 3 (2nd ed.; pp 1643-1647). Oxford, England: Elsevier Sciences, Ltd.

Christian, K, Morrison, FJ, \& Bryant, FB. 1998. Predicting kindergarten academic skills: Interactions among child care, maternal education, and family literacy environments. Early Childhood Research Quarterly.13, 501-521.

Committee on Early Childhood Pedagogy. 2000. Eager to learn: Educating our preschoolers. The National Academies Press; Washington, DC.

Couch, A., \& Kenston, K. (1960). Yeasayers and naysayers: Agreeing response set as a personality variable. Journal of Abnormal and Social Psychology, 60, 151-174.

Darling, N. 1999. Parenting style and its correlates. Clearing house on Elementary and Early Childhood Education. Retrieved from http: //ecap. crc. illinois. edu/ eecearchive/ digests/ 1999/ darlin99.pdf

Darling.N, \& Steinberg, L. 1993. Parenting style as context: An integrative model. Psychological Bulletin, 113 (3), 487-496.

Elias, MS. 2006. Relationship between parent-child interaction and academic performance of the child. Journal of Life and Earth Science, 1(2), 73-75.

Epstein, JL. 1989. Family structure and student motivation: A development perspective. Research on Motivation in Education, 3, 259-295.

Estrada, P, Arsenio, WF, Hess, RD, \& Holloway, SC. 1987. Affective quality of the mother-child relationship: Longitudinal consequences for children's school relevant cognitive functioning. Developmental Psychology, 23(2), 210-215.

Fletcher, AC, Walls, JK, Cook, EC, Madison, KJ, \& Bridges, TH. 2008. Parenting style as a moderator of associations between maternal disciplinary strategies and child well-being, Journal of Family Issues, 29 (12), 1724-1744.

Good, V. 1993. Dictionary of Psychology, 3rd Ed., McGraw Hill Book Company.

Hoover-Dempsey, KV, \& Sandler, HM. 1997. Why do parents become involved in their children's education?, Review of Educational Research, 67, 3-42.

Jeynes, W. 2003. A Meta-Analysis: The Effects of Parental Involvement on Minority Children's Academic Achievement. Education \& Urban Society, 35 (2), 202-218.

Kagan, J. 1974. The psychological requirements for human development. In N. B. Talbot (Ed.), Raising children in modern America: Problems and prospective solutions. (pp. 86-97). Boston, MA: Little, Brown, \& Co.

Kagan, J. 1978. The parental love trap. Psychology Today, 12, 54-61.

Kamble,VS, \& Adsul, R. 2012. Academic Performance as Function of Parental Acceptance and Academic Climate among Adolescents. IPEDR, 53 (12), 55-58. 
Karavasilis, L, Doyle, AB, \& Markiewicz, D. 2003. Associations between parenting style and attachment to mother in middle childhood adolescence. International Journal of Behavioral Development, 27(2), 153-164.

Kardiner, A.1939. The Individual and his society. New York: Columbia University Press.

Kardiner, A. 1945a. The concept of basic personality structure as an operational tool in the social sciences. In R. Linton (Ed.) The Science of man in the world crisis (pp. 102-122). N.Y.: Viking Fund.

Kardiner, A. 1945b. The Psychological frontiers of society. New York: Columbia University Press.

Kazmi, SF, Sajjid, M, \& Pervez, T. 2011. Parental style and academic achievement among the students. International Journal of Academic Research, 3(2), 582-588.

Khaleque, A \& Rohner, RP. 2002. Perceived parental acceptancerejection and psychological Adjustment: A meta-analysis of cross-cultural and intercultural studies. Journal of Marriage and Family, 4, 54-64.

Khan, S, Haynes, L, Armstrong, A, \& Rohner, RP. 2010. Perceived teacher acceptance, parental acceptance, academic achievement, and school conduct of middle school students in the Mississippi Delta Region of the United States. Cross-Cultural Research, 44(3), 283-294.

Kordi, A, \& Baharudin, R. 2010. Parenting Attitude and Style and Its Effect on Children's School Achievements, International Journal of Psychological Studies, 2(2), 217-222.

Luby, JL, Barch, DM, Belden, A, Gaffrey, MS, Tillman, R, Babb, C, Nishino, T, Suzuki, H, \& Botteron, KN. 2012. Maternal support in early childhood predicts larger hippocampal volumes at school age. Proceedings of the National Academy of Sciences, 8, 2854-2859.

McClelland, K. 1990. "Cumulative Disadvantage among the Highly Ambitious.' Sociology of Education, 63,102-21.

Mullah, MAS, Parveen, N, Ahshanullah, M. 2007. Tribal people and their socio-economic characteristics in Rangamati sadar thana. BRAC University Journal, 4(1), 47-58.

Phinney, JS. 1990. Ethnic Identity in Adolescents and Adults: Review of Research. Psychological Bulletin, 18 (3), 499-514.

Prindle, C, \& Rasinski, KA. 1989. The National Education Longitudinal Study of 1988: Data collection results and analysis potential. Paper presented at the Annual Meeting of the American Educational Research Association. (ERIC Document Reproduction Service No.: ED 308-215).

Rayan, BA, \& Admas, GR. 1998. Family processes, parent-child interactions and child characteristics influencing school-based social adjustment. Journal of Marriage and the family, 60, 374-37.

Rohner, RP \& Khaleque, A. 2006. Handbook for the study of parental acceptance and rejection. Storrs:Rohner research Publication.
Rohner, RP, Khaleque, A, \& Cournoyer, DE. 2007. Introduction to parental acceptance- rejection theory, methods, evidence, and implications. Retrieved May 8, 2007 from the University of Connecticut Center for the Study of Interpersonal Acceptance and Rejection website at www.csiar.uconn.edu.

Rohner, RP, Khaleque, A, \& Cournoyer, DE. 2012. Introduction to parental acceptance-rejection theory, methods, evidence, and implications. Retrieved November 16, 2012 from the University of Connecticut Center for the Study of Interpersonal Acceptance and Rejection website at www.csiar.uconn.edu.

Rohner, RP. 2005. Parental Acceptance-Rejection Questionnaire (PARQ): Test manual. In R. P. Rohner \& A. Khaleque (Eds.), Handbook for the Study of Parental Acceptance and Rejection $\left(4^{\text {th }}\right.$ Ed.) (pp. 43-106). Storrs, CT: Rohner Research Publications.

Rohner, RP. 1986. The warmth dimension: Foundations of parental acceptance-rejection theory. Beverly Hills, CA: Sage Publications, Inc. (Available from Rohner Research Publications, Storrs, CT).

Russo, JB, \& Owens, RE. 1982. The development of an observation tool for parent-child interaction. Journal of Speech and Hearing Disorders, 47, 165-173.

Spencer, MB, \& Markstrom-Adams, C. 1990. Identity Processes Among Racial and Ethnic Minority Children in America. Child Development, 61, 290-310.

Stephen, J Ceci, et al. 1997. A Bio-Ecological Model of Human Development in Intelligence. Heredity and Environment, Cambridge University Press.

Topor, DR, Keane, SP, Shelton, TL, \& Calkins, SD. 2010. Parent involvement and student academic performance: A multiple mediational analysis. J Prev Interv Community, 38(3): 183-197.

Uddin, MK. 2011. Parental Warmth and Academic Achieve ment of Adolescent Children. Journal of Behavioural Sciences, 21(1), 1-12.

Van Meter, EJ. 1994. Implementing school-based decision making in Kentucky. NASSP Bull., 78: 61-70.

Viernstein, V, \& Hogan, H. 1975. Parental Personality Factors and Achievement Motivation in

Talented Adolescents, Journal of Youth and Adolescence 4 (2), 187-189.

Whiting, JWM, \& Child, IL. 1953. Child training and personality. New Haven, CT: Yale University Press.

Xitao, F, \& Michael, C. 2001. Parental Involvement and Students' Academic Achievement: A Meta-Analysis, Educational Psychology Review, 13(1), 1-22.

Ziegler, S. 1987. The effects of parent involvement on children's achievement: The significance of home school links. Toranto Board of Education, Ontario.

Manuscript received on 6 October 2013 and revised on 28 November 2013 